\title{
Adherence to MRI Protocol Consensus Guidelines in Multiple Sclerosis Patients at Taif Hospitals
}

\author{
Sultan Alamri \\ Radiological Science Department, College of Applied Medical Sciences, Taif University, Taif city, Saudi Arabia \\ Email address: \\ s.alamri@tu.edu.sa

\section{To cite this article:} \\ Sultan Alamri. Adherence to MRI Protocol Consensus Guidelines in Multiple Sclerosis Patients at Taif Hospitals. International Journal of \\ Medical Imaging. Vol. 8, No. 4, 2020, pp. 102-105. doi: 10.11648/j.ijmi.20200804.16
}

Received: November 7, 2020; Accepted: November 17, 2020; Published: November 24, 2020

\begin{abstract}
Background: multiple sclerosis (MS) is a chronic inflammatory disease that affects the central nervous system (CNS). Its diagnosis is characterized by foci of demyelination, disseminated over time and anatomical space, both clinically and radiologically. Consensus guidelines for the diagnosis of MS have been established to identify the proper sequences and imaging technique. Objective: this study aims to investigate the adherence of Taif hospitals to these consensus guidelines. Method: data were collected from the radiology departments of 3 main hospitals in Taif (King Abdul-Aziz Specialist Hospital, King Faisal Medical Complex, and Al-Hada Hospital for Armed Forces). The standard magnetic resonance imaging (MRI) protocol for MS in each hospital was reviewed using the picture archiving and communication system (PACS). The comparisons were based on the strength of the magnetic field, slice thickness, slice orientation, coverage, and the MR core sequences used. Results: each Taif hospital has its own protocol, which differ from consensus guidelines. The main difference is the hospitals' lack of 3D sequences. Another difference is the lack of inversion recovery prepared T1 sequences. Finally, for follow-up patients, no contrast administration exists in Taif hospitals. Conclusion: this study shows some major differences in the MS protocols among Taif hospitals. This is probably due to poor awareness of the guidelines in the radiology community. Neuroradiologists at Taif hospitals should ensure the recommended protocol is followed.
\end{abstract}

Keywords: Multiple Sclerosis, MRI, Radiology, Taif

\section{Introduction}

Multiple Sclerosis (MS) is a chronic inflammatory disease that affects the central nervous system (CNS). It is characterized by foci of demyelination, disseminated over time and anatomical space [1]. MS is among the most common non-traumatic causes of neurological disabilities in young adults. The onset typically occurs from the ages of 20 to 40 with the peak in the mid-30s [2]. The estimated number of people with MS worldwide has increased from 2.1 million in 2008 to 2.3 million in 2013 [3]. Evidence shows that this increase is due to the heightened incidence of the disease among women, as the female to male ratio is approximately 2:1 [4]. The prevalence of MS varies significantly around the world, with the incidence lower in equatorial regions [3]. In Saudi Arabia, although it has been estimated that there are approximately 30 MS cases per 100,000 people, most available studies are hospital-based and lack regional and national investigations of MS prevalence [5].
The diagnosis of MS is largely based on McDonald Criteria that was established in 2001 [6] and subsequently revised [7-9]. McDonald Criteria incorporate both clinical evaluation and imaging techniques disseminated both in space (DIS) and time (DIT) to provide a faster and more accurate diagnosis. Imaging provides critical information in the diagnosis of MS, especially magnetic resonance imaging (MRI). It is also capable of distinguishing the disease from similar disorders as the white matter lesions increase with age among the general population [10]. This is because, on average, MRI is 5 to 10 times more sensitive to demyelination than other imaging techniques [11]. In addition, MRI is important to monitor the progression of the MS and the response to therapy that has been hampered by inconsistent protocols and poor timing [12]. This fact suggested the need for a standardized protocol that identifies when and how MRI should be used to investigate MS in order to accelerate diagnosis [11]. DIS in imaging is demonstrated by the presence of one or more hyperintense 
lesions in 2 or more brain regions, i.e., cortical, periventricular, juxta-cortical, or infra-tentorial. DIT is demonstrated by the presence of gadolinium-enhanced or non-enhanced lesions in 2 different scans.

Evidence based on expert guidelines of MS imaging were first included in 2003 and followed by several modifications in an attempt to enable early detection, monitoring, and (therefore) management of the disease [13, 14]. Since then, the use of MRI for MS diagnosis has been expanded to facilitate the assessment and monitoring of treatment [10-12, 15]. The aim of this article is to identify Taif hospitals' adherence to the guidelines of MS imaging as described by Traboulsee and his colleagues [12]. This is important to facilitate better diagnosis and treatment.

\section{Methods}

Data were collected from the radiology departments of 3 main hospitals in Taif (King Abdulaziz Hospital, King Faisal Medical Complex, and Al-Hada Hospital for Armed Forces). The standard MRI protocol for MS in each hospital was reviewed using the picture archiving and communication system (PACS). This protocol is typically used with patients who are diagnosed with MS or are clinically suspicious. Therefore, this protocol was reviewed to identify to what extent it adheresx to the standardized MS protocol revised by Traboulsee and his colleagues [12] (see Table 1). The comparisons were based on the strength of the magnetic field, slice thickness, slice orientation, coverage, and the MR core sequences used.

\section{Table 1. The standardized MRI protocol for MS.}

\begin{tabular}{ll}
\hline Field strength & $\geq 1.5 \mathrm{~T}$ \\
Coverage & Whole-brain \\
Section thickness & $\leq 3 \mathrm{~mm}$ \\
Slice orientation & Along the subcallosal line for axial slices \\
& 3D inversion recovery-prepared T1 gradient echo \\
& Gadolinium single dose, 0.1 mmol/kg given for 30 \\
& seconds \\
& 3D sagittal T2WI FLAIR \\
Core sequences & 3D T2WI \\
& 2D axial DWI (5-mm sections, no gap) \\
& 3D FLASH (non-IR prep) post-gadolinium \\
& For follow-up, gadolinium is always recommended \\
& Axial proton attenuation \\
& Pre- or post-gadolinium axial T1 spin-echo (for \\
& chronic black holes) \\
& SWI for identification of central vein within T2 \\
& lesions \\
\hline
\end{tabular}

\section{Result}

The MRI protocols for MS at King Faisal Medical Complex, King Abdul-Aziz Specialist Hospital, and Al-Hada Hospital are shown in Table 2, Table 3, and Table 4, respectively. Firstly, at King Faisal Medical Complex, the magnetic strength of the MR machine is at least $1.5 \mathrm{~T}$ (there are $2 \mathrm{MR}$ machines, $1.5 \mathrm{~T}$ and $3 \mathrm{~T}$ ). The MS protocol is comprised of 8 sequences, and the slice thickness is less than
$2 \mathrm{~mm}$ (as shown in Table 2). The protocol begins with the same protocol of all brain MRI scans (axial T1, T2 and FLAIR, and sagittal T1WI), then 3 more sequences are added (3D sagittal that uses double inversion recovery to null the signal from both CSF and white matter, 3D sagittal FLAIR, and sagittal T2 FLAIR). After the administration of $10 \mathrm{ml}$ of gadolinium, the protocol includes 2 more sequences (axial and sagittal T1). For follow-up cases, the hospital repeats the same protocol but without the administration of contrast media.

Secondly, at King Abdul-Aziz Specialist Hospital, the magnetic strength of the MR machine is $1.5 \mathrm{~T}$ (both MR machines are $1.5 \mathrm{~T}, \mathrm{GE})$. The MS protocol is comprised of 10 sequences, and the slice thickness ranges from 1 to $3.6 \mathrm{~mm}$ (as shown in Table 3). In addition to the routine MRI brain sequences, the protocol in this institution utilizes the magnetic susceptibility sequence (SWAN). Similar to the protocol at King Faisal Medical Complex, follow-up cases here require repetition of the protocol but without the administration of contrast media.

Finally, at Al-Hada Hospital, the magnetic strength of the MR machine is $1.5 \mathrm{~T}$ (both MR machines are $1.5 \mathrm{~T}$, Siemens). The MS protocol is comprised of 10 sequences, and the slice thickness is less than $2 \mathrm{~mm}$ or $5 \mathrm{~mm}$ for DWI sequence (as shown in Table 4). In addition to the routine MRI brain sequences, the protocol in this institution utilizes hemorrhagic GRE pulse sequence (Hemo). Similar to the protocols at King Faisal Medical Complex and King AbdulAziz Specialist Hospital, follow-up cases here require repetition of the protocol but without the administration of contrast media. In addition, all 3 hospitals implement 2D sequences with varied slice gaps and brain coverage from the foramen magnum to the superior border of the head. Moreover, the orientation of the axial slices is across the anterior-posterior commissure axis or just parallel with the genu and splenium of the corpus callosum.

Table 2. The MS protocol at King Faisal Medical Complex.

\begin{tabular}{ll}
\hline Field strength & $1.5 \mathrm{~T}$ and $3 \mathrm{~T}$ \\
Slice thickness & $<2 \mathrm{~mm}$ \\
Coverage & Base to vertex of brain \\
& Axial T1 WI \\
& Axial T2WI and FLAIR \\
& Sagittal T1 WI \\
& Axial DWI \\
& In case of detection of MS they add 3 more sequences: \\
Core & Sagittal CUBE DIR WM Null \\
Sequences & Sagittal CUBE FLAIR \\
& Sagittal T2 FLAIR \\
& Axial and sagittal T1 post contrast \\
& Follow-up cases they use the same protocol except \\
& without contrast media \\
\hline
\end{tabular}

Table 3. The MS protocol at King Abdulaziz Specialist Hospital.

\begin{tabular}{ll}
\hline Field strength & $1.5 \mathrm{~T}$ \\
Slice thickness & $=3.6 \mathrm{~mm}$ \\
Coverage & Whole brain \\
& 3D axial SWAN \\
Core Sequences & 3D sag. T2 FLAIR -CUBE \\
& Axial DWI \\
\hline
\end{tabular}




\begin{tabular}{ll}
\hline Axial T1 PROPELLER. \\
Axial T2 Flair PROPELLER \\
Axial T2 PROPELLER \\
Coronal T2 PROPELLER \\
Sagittal T1 FLAIR \\
Axial and sagittal T1 post contrast \\
No contrast for follow-up cases \\
\hline
\end{tabular}

Table 4. The MS protocol at Al-Hada Hospital for Armed Forces.

\begin{tabular}{ll}
\hline Field strength & $1.5 \mathrm{~T}$ \\
Slice thickness & $<2 \mathrm{~mm}$ \\
Coverage & Whole brain \\
& Axial DWI (1.5 mm gap and $5 \mathrm{~mm}$ slice thickness) \\
& Hemo T2 axial (GRE) \\
& Axial T2 FSE \\
Core Sequences & Axial Flair \\
& Axial T1 FSE \\
& Sagittal T1 \\
& T1 FSE (axial, sagittal and coronal) post contrast \\
& No contrast for follow-up cases \\
\hline
\end{tabular}

\section{Discussion}

MS is one of the most common neurological diseases. MRI is an essential technique to differentiate between MS and other white matter lesions. Therefore, we aim to investigate the adherence of Taif hospitals to the standardized MRI protocol recommended by Traboulsee and his colleagues [16]. The results of this study show that adherence to the standardized protocol varies among the hospitals. First of all, in terms of magnetic field, all 3 hospitals' machines are at least $1.5 \mathrm{~T}$. This is important as the magnetic field strength affects the signal intensity and, thus, the quality of the image. Higher magnetic fields (greater than 1.5T) would be superior in terms of image quality and ability to detect signal abnormalities [17-19]. Stronger magnetic fields have drawbacks, however, such as the high cost and associated risks. Secondly, the brain coverage and slice orientation in each hospital follow the recommended protocol. The importance of this is that the radiologist can detect changes over time.

Furthermore, although the MRI scanners are advanced and capable of producing 3D images, the protocols in all 3 hospitals tend to favor 2D images. This is problematic since 2D images feature slice gaps. The main reason for this tendency might be that $3 \mathrm{D}$ images take more time to complete compared with 2D images, which may present an issue when treating a large number of patients and processing long waiting lists. However, because 3D images have a higher sensitivity for MS lesions in the periventricular region, deep white matter, juxta-cortical region, and infratentorial region [20, 21], they are always recommended in MR protocol.

In addition, sequences in the Taif hospitals' protocol differ greatly from the recommended protocol. First of all, we noticed that the protocols at all Taif hospitals have a sagittal T1WI sequence that is not mentioned in the recommended protocol. This sequence might be necessary, given the lack of 3D sequences in Taif hospitals. Secondly, a susceptibility weighting sequence in the MR protocol is recommended to increase the chance of detecting white matter lesions [22]. This sequence is absent at King Faisal Medical Complex and has been replaced by double inversion recovery to null signals coming from the white matter. Thirdly, 3D inversion recovery prepared $\mathrm{T} 1$ gradient echo is absent in the protocols at Taif hospitals. This sequence is important for volumetric measurements as well as the detection of lesions in the juxtacortical and infratentorial regions [12, 23]. Fourthly, the sagittal T2WI FLAIR sequence recommended by the standardized MS protocol is absent at Al-Hada Hospital. This should be reconsidered since lesions in the corpus callosum and juxta-cortical region are better visualized in the sagittal plane [12]. Finally, it is always recommended to administer gadolinium contrast to MS patients (whether for a new diagnosis or follow-up) to detect new brain lesions and to rule out tumors and leptomeningeal diseases, for example [24]. Unfortunately, this is not applied in all Taif hospitals, as only suspected cases are given the contrast agent.

\section{Conclusion}

In summary, because the diagnosis of MS is based on clinical symptoms and radiological findings, a suitable MRI protocol recommended by experts in the field should be followed. This study shows some major differences in the MS protocols among Taif hospitals. We have also shown that the adherence to MS protocol is inadequate, particularly related to $3 \mathrm{D}$ sequences. This is probably due to the radiology community's poor awareness of the guidelines. Neuroradiologists at Taif hospitals should ensure that recommended protocol is followed. A further study could assess the level of knowledge and awareness of neuroradiologists regarding such matters.

\section{References}

[1] Compston A, Coles A. Multiple sclerosis. Lancet Lond Engl 2002; 359: 1221-31. https://doi.org/10.1016/S01406736(02)08220-X.

[2] Brownlee WJ, Hardy TA, Fazekas F, Miller DH. Diagnosis of multiple sclerosis: progress and challenges. The Lancet 2017; 389: 1336-46. https://doi.org/10.1016/S0140-6736(16)30959$\mathrm{X}$.

[3] Browne P, Chandraratna D, Angood C, Tremlett H, Baker C, Taylor BV, et al. Atlas of Multiple Sclerosis 2013: A growing global problem with widespread inequity. Neurology 2014; 83: 1022-4. https://doi.org/10.1212/WNL.0000000000000768.

[4] Trojano M, Lucchese G, Graziano G, Taylor BV, Jr SS, Lepore V, et al. Geographical variations in sex ratio trends over time in multiple sclerosis. PLoS ONE 2012; 7: e48078. https://doi.org/10.1371/journal.pone.0048078.

[5] Heydarpour P, Khoshkish S, Abtahi S, Moradi-Lakeh M, Sahraian MA. Multiple Sclerosis Epidemiology in Middle East and North Africa: A Systematic Review and MetaAnalysis. Neuroepidemiology 2015; 44: 232-44. https://doi.org/10.1159/000431042. 
[6] McDonald WI, Compston A, Edan G, Goodkin D, Hartung H$\mathrm{P}$, Lublin FD, et al. Recommended diagnostic criteria for multiple sclerosis: Guidelines from the international panel on the diagnosis of multiple sclerosis. Ann Neurol 2001; 50: 1217. https://doi.org/10.1002/ana.1032.

[7] Polman CH, Reingold SC, Edan G, Filippi M, Hartung H-P, Kappos L, et al. Diagnostic criteria for multiple sclerosis: 2005 revisions to the "McDonald Criteria." Ann Neurol 2005; 58: 840-6. https://doi.org/10.1002/ana.20703.

[8] Thompson AJ, Banwell BL, Barkhof F, Carroll WM, Coetzee T, Comi G, et al. Diagnosis of multiple sclerosis: 2017 revisions of the McDonald criteria. Lancet Neurol 2018; 17: 162-73. https://doi.org/10.1016/S1474-4422(17)30470-2.

[9] Polman CH, Reingold SC, Banwell B, Clanet M, Cohen JA, Filippi M, et al. Diagnostic criteria for multiple sclerosis: 2010 Revisions to the McDonald criteria. Ann Neurol 2011; 69: 292-302. https://doi.org/10.1002/ana.22366.

[10] Filippi M, Rocca MA, Ciccarelli O, De Stefano N, Evangelou N, Kappos L, et al. MRI criteria for the diagnosis of multiple sclerosis: MAGNIMS consensus guidelines. Lancet Neurol 2016; 15: 292-303. https://doi.org/10.1016/S14744422(15)00393-2.

[11] Simon JH, Li D, Traboulsee A, Coyle PK, Arnold DL, Barkhof F, et al. Standardized MR imaging protocol for multiple sclerosis: Consortium of MS Centers consensus guidelines. AJNR Am J Neuroradiol 2006; 27: 455-61.

[12] Traboulsee A, Simon JH, Stone L, Fisher E, Jones DE, Malhotra A, et al. Revised Recommendations of the Consortium of MS Centers Task Force for a Standardized MRI Protocol and Clinical Guidelines for the Diagnosis and Follow-Up of Multiple Sclerosis. AJNR Am J Neuroradiol 2016; 37: 394-401. https://doi.org/10.3174/ajnr.A4539.

[13] Tintoré M, Rovira A, Río J, Nos C, Grivé E, Sastre-Garriga J, et al. New diagnostic criteria for multiple sclerosis: application in first demyelinating episode. Neurology 2003; 60: 27-30. https://doi.org/10.1212/wnl.60.1.27.

[14] Miller DH, Filippi M, Fazekas F, Frederiksen JL, Matthews PM, Montalban X, et al. Role of magnetic resonance imaging within diagnostic criteria for multiple sclerosis. Ann Neurol 2004; 56: 273-8. https://doi.org/10.1002/ana.20156.

[15] Rovira À, Wattjes MP, Tintoré M, Tur C, Yousry TA, Sormani MP, et al. Evidence-based guidelines: MAGNIMS consensus guidelines on the use of MRI in multiple sclerosis-clinical implementation in the diagnostic process. Nat Rev Neurol 2015; 11: 471-82. https://doi.org/10.1038/nrneurol.2015.106.

[16] Traboulsee A, Simon JH, Stone L, Fisher E, Jones DE, Malhotra A, et al. Revised recommendations of the consortium of MS centers task force for a standardized MRI protocol and clinical guidelines for the diagnosis and followup of multiple sclerosis. Am J Neuroradiol 2015.

[17] Di Perri C, Dwyer MG, Wack DS, Cox JL, Hashmi K, Saluste E, et al. Signal abnormalities on 1.5 and 3 Tesla brain MRI in multiple sclerosis patients and healthy controls. A morphological and spatial quantitative comparison study. NeuroImage 2009; 47: 1352-62. https://doi.org/10.1016/j.neuroimage.2009.04.019.

[18] Fischbach F, Bruhn H. Assessment of in vivo $1 \mathrm{H}$ magnetic resonance spectroscopy in the liver: a review. Liver Int 2008; 28: 297-307. https://doi.org/10.1111/j.14783231.2007.01647.x.

[19] Wattjes MP, Harzheim M, Lutterbey GG, Hojati F, Simon B, Schmidt S, et al. Does high field MRI allow an earlier diagnosis of multiple sclerosis? J Neurol 2008; 255: 1159-63. https://doi.org/10.1007/s00415-008-0861-3.

[20] Patzig M, Burke M, Brückmann H, Fesl G. Comparison of 3D cube FLAIR with 2D FLAIR for multiple sclerosis imaging at 3 Tesla. ROFO Fortschr Geb Rontgenstr Nuklearmed 2014; 186: 484-8. https://doi.org/10.1055/s-0033-1355896.

[21] Tawfik AI, Kamr WH. Diagnostic value of 3D-FLAIR magnetic resonance sequence in detection of white matter brain lesions in multiple sclerosis. Egypt J Radiol Nucl Med 2020; 51: 127. https://doi.org/10.1186/s43055-020-00247-6.

[22] Clarke MA, Pareto D, Pessini-Ferreira L, Arrambide G, Alberich M, Crescenzo F, et al. Value of 3T SusceptibilityWeighted Imaging in the Diagnosis of Multiple Sclerosis. Am J Neuroradiol 2020. https://doi.org/10.3174/ajnr.A6547.

[23] Ma Y-J, Jang H, Wei Z, Cai Z, Xue Y, Lee RR, et al. Myelin Imaging in Human Brain Using a Short Repetition Time Adiabatic Inversion Recovery Prepared Ultrashort Echo Time (STAIR-UTE) MRI Sequence in Multiple Sclerosis. Radiology 2020; 297: 392-404. https://doi.org/10.1148/radiol.2020200425.

[24] Granziera C, Reich DS. Gadolinium should always be used to assess disease activity in MS - Yes. Mult Scler Houndmills Basingstoke Engl 2020; 26: 765-6. https://doi.org/10.1177/1352458520911174. 